\title{
Resistance Coefficients of Polymer Membrane with Concentration Polarization
}

\author{
Andrzej Ślęzak • Sławomir Grzegorczyn • \\ Kornelia M. Batko
}

Received: 9 October 2011 / Accepted: 19 June 2012 / Published online: 25 July 2012

(C) The Author(s) 2012. This article is published with open access at Springerlink.com

\begin{abstract}
The Kedem-Katchalsky equations, modified by means of symmetric transformations of Peusner thermodynamic networks, were applied to interpret the membrane transport in concentration polarization conditions. The results from the study demonstrate that the resistance coefficients counted for membrane transport of aqueous solutions of glucose through Nephrophan membrane in horizontal plane are nonlinearly dependent on mean concentration of glucose in the membrane $(\bar{C})$. It was also shown that the threshold value of concentration $\left(\bar{C}_{c r}\right)$ existed, and for $\bar{C}>\bar{C}_{c r}$, the resistance coefficients depend, while for $\bar{C}<\bar{C}_{c r}$, they do not depend on the membrane system configuration. Increase of mean glucose concentration in the membrane (in the range $\bar{C}>\bar{C}_{c r}$ ) causes decrease of difference between resistance coefficients of the membrane system in homogeneous conditions (solutions mechanically stirred) and in conditions with hydrodynamic instabilities (configuration B). Besides increase of mean glucose concentration in the membrane (in the range $\bar{C}>\bar{C}_{c r}$ ) causes increase of the difference between resistance coefficients for membrane system with concentration polarization without hydrodynamic instabilities (configuration A) and membrane system in homogeneous conditions.
\end{abstract}

Keywords Membrane transport · Peusner's network thermodynamics · Kedem-Katchalsky equations $\cdot$ Concentration polarization $\cdot$ Resistance coefficients

\footnotetext{
A. Ślęzak

Institute of Public Health, Department of Biophysics, Częstochowa University of Technology, 36b Armia Krajowa Al., 42200 Czestochowa, Poland

e-mail: aslezak52@gmail.com

S. Grzegorczyn $(\bowtie)$

Department of Biophysics, Silesia Medical University, 16 H. Jordan Str., 41808 Zabrze, Poland e-mail: grzegorczyn@sum.edu.pl

K. M. Batko

Department of Informatics for Economics, University of Economics in Katowice, 2B Bogucicka Str., 40287 Katowice, Poland

e-mail: korabatko@ue.katowice.pl
} 


\section{List of Symbols}

$R_{i j} \quad$ Generalized resistance coefficients

$X_{i} \quad$ Thermodynamic forces in homogeneous conditions

$J_{i} \quad$ Thermodynamic fluxes in homogeneous conditions

$X_{i}^{*} \quad$ Thermodynamic forces in non-homogeneous conditions

$J_{i}^{*} \quad$ Thermodynamic fluxes in non-homogeneous conditions

$J_{v} \quad$ Volume flux in homogeneous conditions

$J_{v s} \quad$ Volume flux in non-homogeneous conditions

$J_{s} \quad$ Solute flux in homogeneous conditions

$J_{s s} \quad$ Solute flux in non-homogeneous conditions

$J_{s a} \quad$ Solute advective flux

$L_{p} \quad$ Hydraulic permeability coefficient

$\sigma \quad$ Reflection coefficient

$\omega \quad$ Solute permeability coefficient

$v \quad$ Kinematic viscosity

$\delta_{k} \quad$ Thickness of concentration boundary layers

$P_{h}, P_{l} \quad$ Hydrostatic pressure ( $h$ higher and $l$ lower value)

$\pi \quad$ Osmotic pressure

$C_{h}, C_{l}$ Solute concentrations in chambers of the membrane system

$\bar{C} \quad$ Mean solute concentration in the membrane

$R \quad$ Gas constant

$R_{C} \quad$ Concentration Rayleigh number

$T \quad$ Thermodynamic temperature

$D_{k} \quad$ Diffusion coefficient

$\zeta_{p} \quad$ Hydraulic concentration polarization coefficient

$\zeta_{v} \quad$ Osmotic concentration polarization coefficient

$\zeta_{s} \quad$ Diffusive concentration polarization coefficient

$\zeta_{a} \quad$ Advective concentration polarization coefficient

\section{Introduction}

Biophysical systems, because of its complex structure, organization and numerous couplings, show nonlinear properties implying their unique behaviour, especially in living organisms (Oster et al. 1971; Nicolis and Prigogine 1977; Peusner 1970). For this reason, the analysis of biophysical systems cannot be reduced to investigations of linear thermodynamic systems (Demirel 2002; Newman and Forgacs 2005; Grzegorczyn et al. 2008). A few of the methods of searching of practical formulations, sufficient and indispensable to analyse such systems, were the attempts to connect thermostatic, nonlinear thermodynamics theory of electrical circuits, topology and chemical kinetics (Meixner 1966; Peusner 1970; Oster et al. 1971; Perelson 1975; Peusner 1986a). The Network Thermodynamics was born in such conditions.

Aharon Katchalsky, Georg Oster and Alan Perelson (Oster et al. 1971) elaborated the term Network Thermodynamics (NT) and its general principles such as basic equations and symbols. NT is the synthesis of non-equilibrium thermodynamics, theory of electric circuits, theory of graphs and differential geometry. NT allows us to describe topology of the system and enables us to analyse dynamics of non-equilibrium processes of mass, charge, energy and information transport (Oster et al. 1971; Perelson 1975; Peusner 1986a; Mikulecky 2005). 
Practical application of NT is based on bond graph method elaborated by Playtner (Playtner 1961) and was introduced to thermodynamics by Oster, Perelson and Katchalsky (Oster et al. 1971) and additionally by Peusner (1970; 1983; 1985a; 1985b, 1986). Peusner's method uses non-equilibrium thermodynamics and theory of electrical circuits and it was shown that both methods are equivalent (Peusner 1986). The NT has been applied to phenomenological description of different kinds of dynamical systems (Imai 1996, 2003; Mikulecky 2005) and was also used in various scopes of biophysics, biochemistry, electrochemistry or chemical engineering (Imai 1996, 2003; Peusner et al. 1985; Newman and Forgacs 2005). The detailed description of the NT was presented in several articles (Perelson 1975; Peusner 1986b Imai 1996; Mikulecky 2005).

The idea of Peusner Network Thermodynamics was presented in 1970 (Peusner 1970) and next developed and used in descriptions of systems converting of energy (Peusner 1983), membrane systems and processes (Peusner 1983), Brown motions (Peusner 1985b) and chemical reaction with diffusion (Peusner et al. 1985). In 1983, (Peusner 1983) Peusner introduced the parameter Q (energy coupling parameter) which has been applied to study efficiency and stability of physical and biological systems, conversion of energy. Besides, the ways of transformations of linear Onsager's equations by means of symmetric and hybrid descriptions were elaborated by Peusner (Peusner 1983, 1985a), and the manners of derivation of Kedem-Katchalsky equations by series of net transformations were also shown.

The assumption of homogeneous solutions, realized in physico-chemical macrosystems by intensive stirring of solutions, diminishes the effect of concentration polarization of a membrane. Description of the influence of concentration polarization on the values of resistance coefficients resulting from PNT was the aim of this article. The resistance coefficients $R_{11}, R_{12}, R_{21}$ and $R_{22}$, determined for homogeneous solutions, are compared (for the same value of mean concentration of glucose in the membrane) with coefficients $R_{11}^{*}, R_{12}^{*}, R_{21}^{*}$ and $R_{22}^{*}$, determined for polymer membrane in conditions of concentration polarization. All of above mentioned coefficients were determined for aqueous glucose solutions and hemodialysis membrane (Nephrophan), and two configurations of the membrane system with membrane in horizontal plane, and glucose solutions with higher concentration under the membrane (configuration A) and over the membrane (configuration B).

\section{The Resistance Coefficients of a Membrane in Concentration Polarization Conditions}

Let us consider the single-membrane system shown in Fig. 1, in which a neutral, symmetric, isotropic and selective polymer membrane (M) separates two heterogeneous (mechanically unstirred) binary non-electrolyte solutions with concentrations $C_{h}$ and $C_{l}\left(C_{h}>C_{l}\right)$ at the initial moment $(t=0)$. In the configuration $\mathrm{A}$, the solution with concentration $C_{l}$ is located in the compartment above the membrane, whereas solution with concentration $C_{h}$ is located below the membrane. In the configuration $\mathrm{B}$, the locations of solutions with concentrations $C_{l}$ and $C_{h}$ are the opposite.

Under these conditions, water and dissolved substance diffusing through the membrane form the CBLs, $l_{\mathrm{h}}$ and $\mathrm{l}_{\mathrm{l}}$, on both sides of the membrane with thicknesses $\delta_{h}$ and $\delta_{l}$, respectively. These layers cause the decrease, of concentration from $C_{h}$ to $C_{i}$ at the membrane surface in $\mathrm{CBL}_{\mathrm{h}}$ and increase from $C_{l}$ to $C_{e}$ at the membrane surface in CBL $l_{l}$. When the solution of lower density is below the membrane and difference of concentration between chambers in the membrane system is sufficiently high, then the system $\mathrm{l}_{\mathrm{h}} / \mathrm{M} / \mathrm{l}_{1}$ loses stability, and natural convection may appear in the near-membrane areas (Ślęzak et al. 1985; Kargol 
a

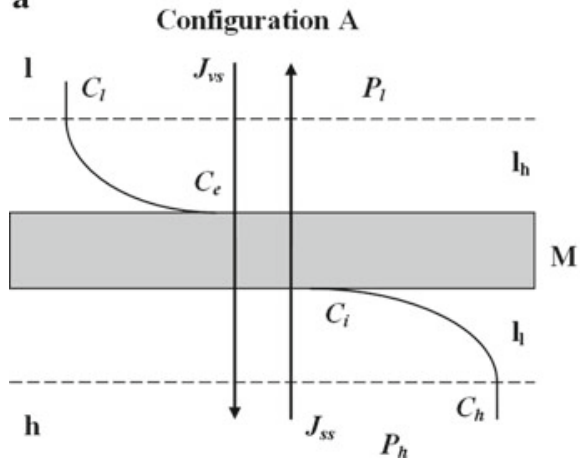

b

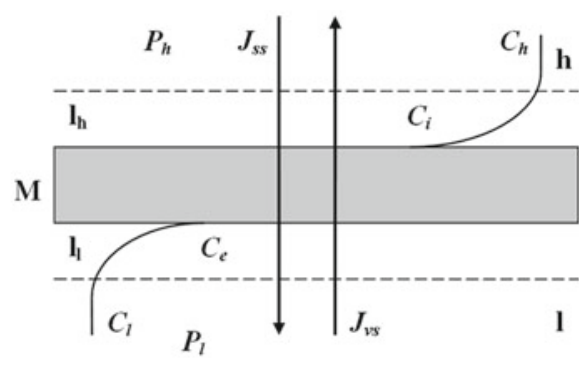

Fig. 1 The membrane system: $M$ membrane; $1_{l}$ and $1_{h}$ the concentration boundary layers (CBLs), $P_{h}$ and $P_{l}$ mechanical pressures; $C_{l}$ and $C_{h}$ concentrations of solutions outside the boundaries, respectively; $C_{e}$ and $C_{i}$ the concentrations of solutions at boundaries, $1_{l} / \mathrm{M}$ and $\mathrm{M} / 1_{h}$, respectively; $J_{v m}$ the volume fluxes through membrane $\mathrm{M} ; J_{v s}$ the volume fluxes through complex $1_{l} / \mathrm{M} / 1_{h} ; J_{s l}, J_{s h}$ and $J_{s m}$ the solute fluxes through layers $1_{l}, 1_{h}$ and membrane, respectively; $J_{s s}$ the solute fluxes through complex $1_{l} / \mathrm{M} / \mathrm{l}_{h}$

1999). This process limits the increase of thickness of CBL and accelerates diffusion of substances outside the layers (Ślęzak 1989; Kargol 2000). Natural convection occurs when the hydrodynamic conditions cause the CBL thicknesses $\delta_{h}$ and $\delta_{l}$ to reach their critical values $\left(\delta_{h}\right)_{\text {crit }}$ and $\left(\delta_{l}\right)_{\text {crit }}$, and when the concentration Rayleigh number $\left(R_{C}\right)$ that control the natural convection process also reaches critical value (Ślęzak et al. 2010; Jasik-Ślęzak et al. 2011). Then, the process of natural convection appears and in certain conditions can even lead to the liquid-type structure called 'plum structure' (Dworecki et al. 2005; Puthenveettil and Arakeri 2008). The process of creation of concentration layers may be demonstrated by optical methods (Dworecki 1995; Dworecki et al. 2005). In the special case, when the process of creation of the layers $l_{\mathrm{h}}$ and $\mathrm{l}_{\mathrm{l}}$ is symmetrical, one can assume that $\delta_{h}=\delta_{l}=\delta$.

The membrane $(\mathrm{M})$ is characterized by the hydraulic permeability called mechanical filtrations $\left(L_{p}\right)$, reflection $(\sigma)$ and solute permeability $(\omega)$ coefficients. The layers $l_{l}$ and $l_{\mathrm{h}}$ are characterized by the solute permeabilities $\left(\omega_{l}, \omega_{h}\right)$ and reflection $\left(\sigma_{l}=\sigma_{h}=0\right)$ coefficients, respectively. The solute permeability coefficient of complex $l_{1} / \mathrm{M} / \mathrm{l}_{\mathrm{h}}$ is denoted by $\omega_{s}$. The diffusion coefficients in layers $l_{l}$ and $l_{\mathrm{h}}$ are denoted by $D_{l}$ and $D_{h}$, respectively. The following relation between coefficients: $\omega_{l}, \omega, \omega_{h}$ and $\omega_{s}$ is fulfilled $\omega_{l}^{-1}+\omega^{-1}+\omega_{h}^{-1}=\omega_{s}^{-1}$, where $\omega_{l}=D_{l}\left(R T \delta_{l}\right)^{-1}$ and $\omega_{h}=D_{h}\left(R T \delta_{h}\right)^{-1}$ (Katchalsky and Curran 1965).

The process of creation of CBLs is accompanied by osmotic volume and solute flows, which are measured as the osmotic volume flux $\left(J_{v}\right)$ and solute flux $\left(J_{S}\right)$. We assume that $J_{v}$ and $J_{s}$ are positive when they are directed vertically upwards, and negative when directed downwards. At the initial moment, when solutions separated by the membrane are homogeneous, the fluxes $J_{v}$ and $J_{s}$ are maximal (Ślęzak 1989; Ślęzak et al. 2002), and then the formation of CBLs $l_{\mathrm{h}}$ and $\mathrm{l}_{\mathrm{l}}$ reduces the value of the volume flux from $J_{v}$ to $J_{v s}$ and the value of the solute flux from $J_{S}$ to $J_{S S}$ (Ślęzak 1989).

In the case of homogeneous solutions, gradients of thermodynamic forces are observed only in a membrane, what is an idealized situation. In a real situation, the diffusive layers are built up on both sides of the membrane (Katchalsky and Curran 1965; Barry and Diamond 1984; Ślęzak 1989; Larchet et al. 2008; Mishchuk 2010). Diffusive layers are the cause of lower gradients of thermodynamic forces in the membrane and appearance of substance gradients in diffusive layers at membrane surfaces. In some hydrodynamically well-founded conditions, diffusive layers are partly destroyed by other processes such as free convection, 
for example (Dworecki et al. 2005). The source of resistance coefficients $R_{i j}$ is the phenomenological Onsager equation, connecting thermodynamic forces $\left(X_{i}\right)$ and fluxes $\left(J_{i}\right)$ which can be written as

$$
X_{i}=\sum_{i} R_{i j} J_{j}
$$

where $R_{i j}$ are generalized resistance coefficients. The Eq. (1) is valid for homogeneous solutions divided by a membrane and requires fulfilment of relations $R_{i j}=R_{j i}$. In real conditions, with concentration polarization of a membrane, the Eq. (1) can be written in the form (Ślęzak 2011):

$$
\left[\begin{array}{l}
X_{1} \\
X_{2}
\end{array}\right]=\left[R^{*}\right]\left[\begin{array}{l}
J_{1}^{*} \\
J_{2}^{*}
\end{array}\right]
$$

where

$$
\left[R^{*}\right]=\left[\begin{array}{ll}
R_{11}^{*} & R_{12}^{*} \\
R_{21}^{*} & R_{22}^{*}
\end{array}\right]
$$

Thermodynamic forces $X_{1}$ and $X_{2}$ are the same as in homogeneous conditions. In this case the symmetry relation $R_{12}^{*}=R_{21}^{*}$ in Eq. (2) is not necessary.

Equation (2) can be applied to derive Kedem-Katchalsky equations by means of transformations of the thermodynamic networks, similarly as in the Peusner's article (Peusner 1983, 1985a). In the case of concentration polarization conditions, the Kedem-Katchalsky equations can be written as

$$
\begin{aligned}
J_{v s} & =\zeta_{p} L_{p}\left(\Delta P-\zeta_{v} \sigma \Delta \pi\right) \\
J_{s s} & =\zeta_{s} \omega \Delta \pi+\bar{C}\left(1-\zeta_{a} \sigma\right) J_{v s}
\end{aligned}
$$

where $J_{v s}$ and $J_{s s}$ are the volume and solute fluxes, $L_{p}, \sigma$ and $\omega$ are the coefficients of hydraulic permeability, reflection and solute permeability, respectively. $\Delta P=P_{h}-P_{l}$ is the difference of hydrostatic pressure $\left(P_{h}\right.$ and $P_{l}$ mean higher and lower values of hydrostatic pressure, respectively); $\Delta \pi=R T\left(C_{h}-C_{l}\right)$ is the difference in osmotic pressures $(R T$ means product of gas constant and thermodynamic temperature, while $C_{h}$ and $C_{l}$ are the solute concentrations in chambers of the membrane system). $\bar{C}=\left(C_{h}-C_{l}\right)\left[\ln \left(C_{h} C_{l}^{-1}\right)\right]^{-1}$ is the mean solute concentration in the membrane. It should be stressed that the numerical values of coefficients $L_{p}, \sigma$ and $\omega$ can be determined in a series of independent experiments (Katchalsky and Curran 1965). The coefficients $\zeta_{p}=L_{p s} / L_{p}, \zeta_{v}=\sigma_{v} / \sigma, \zeta_{s}=\omega_{s} / \omega$ and $\zeta_{a}=\sigma_{s a} / \sigma$ are, respectively, (the coefficients of hydraulic, osmotic, diffusive and advective concentration polarization, and $L_{p s}, \sigma_{s}, \omega_{s}$, and $\sigma_{s a}$ are correspondingly the coefficients of hydraulic permeability, reflection, solute permeability and reflection-advective for complex: membrane and CBLs. Equation (3) describes volume flux of solution; the first component of Eq. (3): $\zeta_{p} L_{p} \Delta P=J_{v s h}$, concerns the hydraulic volume flux, and the second: $\zeta_{p} L_{p} \zeta_{v} \sigma \Delta \pi=J_{v s o}$, the osmotic volume flux. Then, Eq. (4) describes solute flux. It is worth to note that the first component of Eq. (4), $\zeta_{s} \omega \Delta \pi=J_{s s d}$, describes the diffusive flux, and the second component, $\bar{C}\left(1-\zeta_{a} \sigma\right) J_{v s}=J_{s a}$, the advective flux.

Taking into account the Eq. (3), the Eq. (4) can be written in the form:

$$
J_{s s}=\left[\zeta_{s} \omega-\bar{C}\left(1-\zeta_{a} \sigma\right) \zeta_{p} L_{p} \zeta_{v}\right] \Delta \pi+\bar{C}\left(1-\zeta_{a} \sigma\right) \zeta_{p} L_{p} \Delta P
$$

where $\bar{C}\left(1-\zeta_{a} \sigma\right) \zeta_{p} L_{p}=\omega_{s a}$ is the advective diffusion permeability coefficient for concentration polarization conditions. The definition of coefficients $\zeta_{p}, \zeta_{v}, \zeta_{s}$ and $\zeta_{a}$ can be obtained from Eqs. (3), (4) and (4a): 


$$
\begin{aligned}
\zeta_{p} & =\left(\frac{1}{L_{p}} \frac{J_{v s h}}{\Delta P}\right)_{\Delta \pi=0} \\
\zeta_{v} & =\left(\frac{\Delta P}{\sigma \Delta \pi}\right)_{J_{v s}=0} \\
\zeta_{s} & =\left(\frac{J_{s s d}}{\omega \Delta \pi}\right)_{J_{v}=0}=\frac{1}{\omega}\left[\frac{J_{s s}}{\Delta \pi}+\bar{C}\left(1-\zeta_{a} \sigma\right) \zeta_{p} \zeta_{v}\right]_{\Delta P=0} \\
\zeta_{a} & =\frac{1}{\sigma}\left(1-\frac{J_{s s a}}{\bar{C} J_{v s}}\right)_{\Delta \pi=0}=\frac{1}{\sigma}\left(1-\frac{J_{s s a}}{\bar{C} \zeta_{p} L_{p} \Delta P}\right)_{\Delta \pi=0}
\end{aligned}
$$

It is worth to pay attention to the article (Ginzburg and Katchalsky 1963), where the concept of the coefficient of diffusive permeability $\left(\omega_{S}\right)$ for complex $l_{1} / M / l_{h}$ was introduced $-l_{l}$ and $l_{h}$ are concentration boundary layers, existing near the membrane in solution with lower (l) and higher (h) concentrations, respectively, and $\mathrm{M}$ is the membrane. Analogously, taking into consideration the pairs of coefficients $L_{p s}$ and $L_{p}, \sigma_{s}$ and $\sigma, \sigma_{a}$ and $\sigma$, the coefficients $\zeta_{p}, \zeta_{v}$ and $\zeta_{a}$ can be obtained. In honour of Professor Aharon Katchalsky, we call the coefficients $\zeta_{p}, \zeta_{v}, \zeta_{s}$ and $\zeta_{a}$ suitably as hydraulic, osmotic, diffusive and advective Katchalsky's factors, respectively.

The Eqs. (3) and (4) can be transformed by simple algebraic transformations to the form:

$$
\begin{aligned}
\Delta P-\Delta \pi & =\left(\frac{\bar{C}\left(1-\zeta_{v} \sigma\right)\left(1-\zeta_{a} \sigma\right)}{\zeta_{s} \omega}+\frac{1}{\zeta_{p} L_{p}}\right) J_{v s}-\frac{1-\zeta_{v} \sigma}{\zeta_{s} \omega} J_{s s} \\
\frac{\Delta \pi}{\bar{C}} & =-\frac{1-\zeta_{a} \sigma}{\zeta_{s} \omega} J_{v s}+\frac{1}{\bar{C} \zeta_{s} \omega} J_{s s}
\end{aligned}
$$

The Eqs. (9) and (10), being of the forms of transformed Kedem-Katchalsky's equations for concentration polarization conditions, can be written in the matrix form:

$$
\left[\begin{array}{l}
\Delta P-\Delta \pi \\
\frac{\Delta \pi}{\bar{C}}
\end{array}\right]=\left[R^{*}\right]\left[\begin{array}{l}
J_{v s} \\
J_{s s}
\end{array}\right]
$$

where $\left[R^{*}\right]$ is the matrix of resistance coefficients given by

$$
\left[R^{*}\right]=\left[\begin{array}{cc}
\frac{\zeta_{s} \omega+\zeta_{p} L_{p} \bar{C}\left(1-\zeta_{v} \sigma\right)\left(1-\zeta_{a} \sigma\right)}{\zeta_{p} L_{p} \zeta_{s} \omega} & -\frac{1}{\zeta_{s} \omega}\left(1-\zeta_{v} \sigma\right) \\
-\frac{1}{\zeta_{s} \omega}\left(1-\zeta_{a} \sigma\right) & \frac{1}{\bar{C} \zeta_{s} \omega}
\end{array}\right]
$$

For homogeneous conditions $\left(\zeta_{p}=\zeta_{s}=\zeta_{v}=\zeta_{a}=1\right)$, we get

$$
[R]=\left[\begin{array}{ll}
\frac{\omega+L_{p} \bar{C}(1-\sigma)^{2}}{L_{p} \omega} & -\frac{1}{\omega}(1-\sigma) \\
-\frac{1}{\omega}(1-\sigma) & \frac{1}{\bar{C} \omega}
\end{array}\right]
$$


Equation (11a) contains coefficients $\zeta_{p}, \zeta_{v}, \zeta_{s}$, and $\zeta_{a}$ determining the conditions of concentration polarization. Moreover, the values of coefficients $R_{11}^{*}, R_{12}^{*}, R_{21}^{*}$ and $R_{22}^{*}$ depend (directly or indirectly by coefficients $\zeta_{v}$ and $\zeta_{s}$ ) on the average solution concentration $(\bar{C})$. For coefficients $L_{p}, \sigma$ and $\omega$ the following conditions are fulfilled: $0 \leq L_{p} \leq\left(L_{p}\right)_{\max }, 0$ $\leq \sigma \leq 1$ and $0 \leq \omega \leq \omega_{\max }$. For unselective membrane $L_{p}=\left(L_{p}\right)_{\max }, \sigma=0$ and $\omega=\omega_{\max }$. Taking into consideration the above conditions and Eq. (11a), for unselective membrane we can write, $R_{1\rfloor}^{*}=\left[\zeta_{s} \omega_{\max }+\zeta_{p}\left(L_{p}\right)_{\max } \bar{C}\right]\left[\zeta_{p}\left(L_{p}\right)_{\max } \zeta_{s} \omega_{\max }\right]^{-1}, R_{12}^{*}=-\left(\zeta_{s} \omega_{\max }\right)^{-1}=R_{21}^{*}$ and $R_{22}^{*}=\left(C \zeta_{s} \omega_{\max }\right)^{-1}$. The transport properties of semipermeable membrane are characterized by $L_{p}>0, \sigma=1$ and $\omega=0$. In that case for this membrane $R_{11}^{*}=R_{22}^{*}=+\infty$ and $R_{12}^{*}=R_{21}^{*}=-\infty$. Next, for selective membrane, $L_{p}>0,0<\sigma<1$ and $\omega>0$. For this case, values of coefficients $R_{11}^{*}, R_{12}^{*}, R_{21}^{*}$ and $R_{22}^{*}$ can be calculated by means of Eq. (11a). Moreover, coefficients $R_{11}^{*}, R_{12}^{*}, R_{21}^{*}$ and $R_{22}^{*}$ are mutually related in the following manner:

$$
R_{11}^{*}-\frac{R_{12}^{*} R_{21}^{*}}{R_{22}^{*}}=\frac{1}{\zeta_{p} L_{p}}
$$

Calculating the quotients $R_{i j} / R_{i j}^{*}$ on the basis of the Eq. (11a) and Eq. (11b), we obtain

$$
\begin{aligned}
\frac{R_{11}}{R_{11}^{*}} & =\frac{\zeta_{p} \zeta_{s}\left[\bar{C}(1-\sigma)^{2} L_{p}+\omega\right]}{\bar{C}\left(1-\zeta_{v} \sigma\right)\left(1-\zeta_{a} \sigma\right) \zeta_{p} L_{p}+\zeta_{s} \omega} \\
\frac{R_{12}}{R_{12}^{*}} & =\frac{(1-\sigma) \zeta_{s}}{1-\zeta_{v} \sigma} \\
\frac{R_{21}}{R_{21}^{*}} & =\frac{(1-\sigma) \zeta_{s}}{1-\zeta_{a} \sigma} \\
\frac{R_{22}}{R_{22}^{*}} & =\zeta_{s}
\end{aligned}
$$

The above relations indicate the influence of concentration polarization on the values of resistivity coefficients of the membrane. On the basis of Eqs. (13)-(16), we can write

$$
\frac{R_{11}}{R_{11}^{*}}\left(\frac{R_{22} R_{12}^{*} R_{21}^{*}}{R_{22}^{*} R_{12} R_{21}} \bar{C}(1-\sigma)^{2} \zeta_{p} L_{p}+\omega\right)=\zeta_{p}\left[\bar{C}(1-\sigma)^{2} L_{p}+\omega\right]
$$

The coefficients $R_{i j}$ and $R_{i j}^{*}$ are counted using Eqs. (11a) and (11b), experimental data: $L_{p}, \sigma, \omega$ and coefficients $\zeta_{p}, \zeta_{s}, \zeta_{v}, \zeta_{a}$, calculated from the dependences of volume and solute fluxes measured for different $\Delta P$ and $\Delta \pi$ in homogeneous and non-honogeneous conditions. The values of coefficients $R_{i j}$ and $R_{i j}^{*}$ are the measures of flow resistance for substance in the membrane, while $R_{i j}^{*} / R_{i j}$ are the measures of changes of transport conditions in the membrane system forced by transition from the homogeneous to non-homogeneous conditions (membrane with concentration polarization). The value of $R_{i j}^{*} / R_{i j}$ is in the range $0<R_{i j}^{*} / R_{i j} \leq 1$ and, can be also treated as the measure of the level of non-homogeneities in the membrane system.

\section{Materials and Methods}

In order to determine the coefficients $\zeta_{p}, \zeta_{v}, \zeta_{s}$ and $\zeta_{a}$, experimental studies of volume and solute flows were carried out by means of the measurement set-up that was described in detail in a previous article (Ślęzak et al. 2010). The set-up consisted of two Plexiglas chambers $(1, h)$ separated by a membrane $(\mathrm{M})$ with a surface area of $3.36 \pm 0.2 \mathrm{~cm}^{2}$. Volumes of the 
chambers $(1, \mathrm{~h})$ were the same and equal to $V_{C}=200 \mathrm{~cm}^{3}$. The chamber (h) was coupled with a calibrated pipette, while the chamber (1) was connected to an external reservoir of pure water at the same height as the pipette. The membrane was mounted in a horizontal plane. The experiments were performed using the flat sheet Nephrophan membrane and aqueous solutions of glucose. This membrane is flat, hydrophilic, micro-porous, symmetric, and has electrically neutral polymeric structure. Cellulose octane has a solid, colourless, transparent, and plastic structure, obtained from plant cellulose with acetic anhydride, acetic acid, and sulphuric acid. The diameter of membrane pores is about $2.4 \mathrm{~nm}$, and the thickness of dry membrane is about $20 \mu \mathrm{m}$ (Klinkmann et al. 1969). Parameters of the membrane, i.e. hydraulic permeability $\left(L_{p}\right)$, reflection $(\sigma)$ and solute permeability $(\omega)$ coefficients were determined in accordance with methods described in Katchalsky and Curran (1965). Values of these coefficients were determined under homogeneity conditions of solution separated by the membrane. Their values for the Nephrophan membrane and for diluted and homogeneous (mechanically stirred) aqueous glucose solutions are as follows: $L_{p}=4.9 \times 10^{-12} \mathrm{~m}^{3} \mathrm{~N}^{-1}$ $\mathrm{s}^{-1}, \sigma=0.068$ and $\omega=0.8 \times 10^{-9} \mathrm{~mol} \mathrm{~N}^{-1} \mathrm{~s}^{-1}$ (Ślęzak 1989; Kargol and Kargol 2000).

At the initial moment, chamber $h$ contained aqueous glucose solution with concentration $C_{h}$, and chamber $l$ contained aqueous glucose solution with concentration $C_{l}\left(C_{h}>C_{l}\right)$. The lower glucose concentration amounted to $C_{l}=1 \mathrm{~mol} \mathrm{~m}^{-3}$, while the concentration $C_{h}$ was changing from 2.6 to $121 \mathrm{~mol} \mathrm{~m}^{-3}$ or $C_{h}=C_{l}$ was changing from 2.6 to $101 \mathrm{~mol} \mathrm{~m}^{-3}$. All experiments were carried out at temperature: $T=295 \pm 0.5 \mathrm{~K}$. Coefficient of diffusion for glucose in aqueous glucose solutions equals to $D_{d}=0.69 \times 10^{-9} \mathrm{~m}^{2} \mathrm{~s}^{-1}$.

The volume flux was calculated on the basis of equation, $J_{v}=\Delta V / S \Delta t$, where $S$ is the membrane's surface area, and $\Delta V / \Delta t$ is the volume of solution transported through the membrane $(\Delta V)$ in the time $(\Delta t)$ (Ślęzak 1989). The solute flux was calculated on the basis of equation, $J_{s}=V_{C} \Delta C / S \Delta t$, where $S$ is the membrane's surface area, and $\Delta C / \Delta t$ is the change of concentration $(\Delta C)$ occurring in the time $(\Delta t)$ and $V_{C}$ is the volume of the measuring chamber (Ślęzak 1989). Evaluation of the concentration changes in the solution was performed by optical method (Ewing 1985).

Each experiment was performed for configurations $\mathrm{A}$ and $\mathrm{B}$ of the membrane system. In configuration $\mathrm{A}$, the upper chamber contained aqueous glucose solution at concentration $C_{l}=$ const. $=1 \mathrm{~mol} \mathrm{~m}^{-3}$, and the lower chamber contained aqueous glucose solution at concentration $C_{h}$. For configuration $\mathrm{A}$, the difference of concentration on the membrane was assumed as negative $(\Delta \mathrm{C}<0)$, while for configuration $\mathrm{B}$ as positive $(\Delta \mathrm{C}>0)$. In configuration $\mathrm{B}$, the upper chamber contained aqueous glucose solution at concentration $C_{h}$, whereas the lower one was filled with aqueous glucose solution at concentration $C_{l}=$ const. $=1 \mathrm{~mol} \mathrm{~m}^{-3}$. In order to determine the coefficients $\zeta_{p}, \zeta_{v}, \zeta_{s}$ and $\zeta_{a}$, the measurements of $J_{v}, J_{v h}, J_{s}$ or $J_{s a}$ for both configurations were taken according to the following procedure (Ślęzak 1989). Firstly, these fluxes were measured in the membrane system with solutions stirred mechanically at $500 \mathrm{rpm}$. After achieving the steady state during which solute and volume fluxes were constant, stirring was stopped, and subsequently the evolutions of the volume or solute fluxes were measured up to reaching of the steady state, with constant and lower values of volume and solute fluxes than at the initial moment. For both configurations experimental time dependencies $J_{v}=f(t), J_{v h}=f(t), J_{v h s}=f(t), J_{v s}=f(t), J_{s}=f(t), J_{s a}=f(t)$ and $J_{s s}=f(t)$ were measured. On the basis of above characteristics, the dependencies $J_{v}=f(\Delta C)_{\Delta P=0}, J_{v s}=f(\Delta C)_{\Delta P=0}, J_{s}=f(\Delta C)_{\Delta P=0}, J_{s s}=(\Delta C)_{\Delta P=0}, J_{v h}=$ $f(\Delta P)_{\Delta \pi=0}, J_{v h s}=f(\Delta P)_{\Delta \pi=0}$ and $J_{s a}=f\left(\bar{C} J_{v}\right)_{\Delta \pi=0}\left(\Delta \pi=R T\left(C_{h}-C_{l}\right)=\right.$ $0, C_{h}=C_{l} \neq 0$ ) for steady states were calculated. The coefficients $\zeta_{p}, \zeta_{v}, \zeta_{s}$ and $\zeta_{a}$ were calculated based on Eqs. (5)-(8). 


\section{Results and Discussion}

In Fig. 2, the characteristics of $J_{v h}=f(\Delta P)(\square)$ and $J_{v h s}=f(\Delta P)(\mathrm{O})$ in the conditions of $\Delta \pi=0\left(C_{h}=C_{l}=100 \mathrm{~mol} \mathrm{~m}{ }^{-3}\right)$ are shown. The first characteristic was obtained in homogeneous conditions (during mechanical stirring of solutions in chambers) and the second, in conditions of concentration polarization of the membrane (without stirring of solutions in chambers). Both characteristics shown in Fig. 2 are linear and independent of the membrane system configurations. The values of fluxes $J_{v h}$ and $J_{v h s}$ are within $5 \%$ measurement error range.

Values of volume $\left(J_{v}, J_{v s}\right)$ and solute $\left(J_{s}, J_{s s}\right)$ fluxes for configurations A and B of the single-membrane system were determined experimentally and shown in Figs. 3 and 4. As seen from Figs. 3 and 4, the dependencies $J_{v}=f(\Delta C)$ and $J_{s}=f(\Delta C)$ for solutions in homogeneity condition are linear and independent of configuration of the membrane system. This means that the values of both $J_{v}$ and $J_{s}$ are the same (symmetric) for both gravitational directions. On the other hand, characteristics $J_{v s}=f(\Delta C)$ and $J_{s s}=f(\Delta C)$ for the solutions in concentration polarization conditions are nonlinear and dependent on the membrane system configuration.

In the range of concentration $-15 \mathrm{~mol} \mathrm{~m}^{-3} \leq \Delta C \leq 15 \mathrm{~mol} \mathrm{~m}^{-3}$, the values of volume and solute fluxes correspondingly for both gravitational directions are the same: $\left(J_{v s}\right)_{\mathrm{A}}=\left(J_{v s}\right)_{\mathrm{B}}$ and $\left(J_{s s}\right)_{\mathrm{A}}=\left(J_{s s}\right)_{\mathrm{B}}$. For $\Delta C<-15 \mathrm{~mol} \mathrm{~m}^{-3}$ (configuration A of the membrane system) and for $\Delta C>15 \mathrm{~mol} \mathrm{~m}^{-3}$ (configuration $\mathrm{B}$ of the membrane system): $\left(J_{v s}\right)_{\mathrm{A}}<\left(J_{v s}\right)_{\mathrm{B}}$ and $\left(J_{s s}\right)_{\mathrm{A}}<\left(J_{s s}\right)_{\mathrm{B}}$. The change of transport properties of the singlemembrane system in configuration $\mathrm{B}$ from diffusive and osmotic (for $\Delta C \leq 15 \mathrm{~mol} \mathrm{~m}^{-3}$ ) to diffusive, osmotic and convective (for $\Delta C>15 \mathrm{~mol} \mathrm{~m}^{-3}$ ) (Rubinstein and Zaltzman 2000; Dworecki et al. 2005; Larchet et al. 2008; Puthenveettil and Arakeri 2008; Puthenveettil et al. 2011; Ramareddy and Puthenveettil 2011) is the cause of asymmetry of characteristics, $J_{v s}=f(\Delta C)$ and $J_{s s}=f(\Delta C)$. Relatively greater volume fluxes in configuration B in comparison with configuration $\mathrm{A}$ is caused by emergence of additional osmotic pressure gradients connected with hydrodynamic instabilities of CBLs (Dworecki et al. 2005; Ślęzak et al.

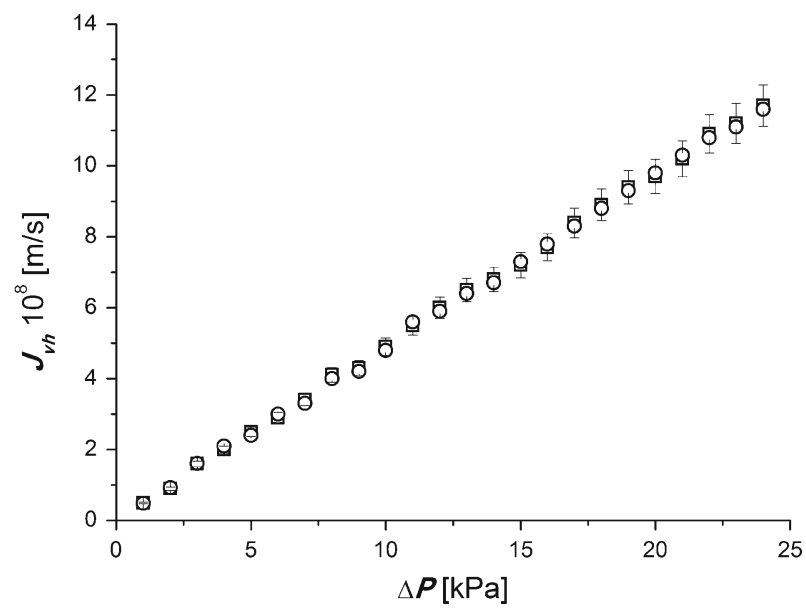

Fig. 2 Hydrostatic pressure difference $\left(\Delta P=P_{h}-P_{l}\right)$ dependence of volume hydraulic flux $\left(J_{v h}\right)$ for the single-membrane system: (open square) $J_{v h}=f(\Delta P)$ in homogeneity solutions conditions; (open circle) $J_{v h s}=f(\Delta P)$ in concentration polarization conditions for $C_{h}=C_{l}=100 \mathrm{~mol} \mathrm{~m}^{-3}$ 




Fig. 3 Glucose concentration difference $\left(\Delta C=C_{h}-C_{l}\right)$ dependence of volume osmotic flux $\left(J_{v}, J_{v s}\right)$ for the single-membrane system: (open square) $J_{v}=f(\Delta C)$ - in homogeneity solutions conditions, (open circle) $J_{v s}=f(\Delta C)$ in concentration polarization conditions for $\Delta P=0$

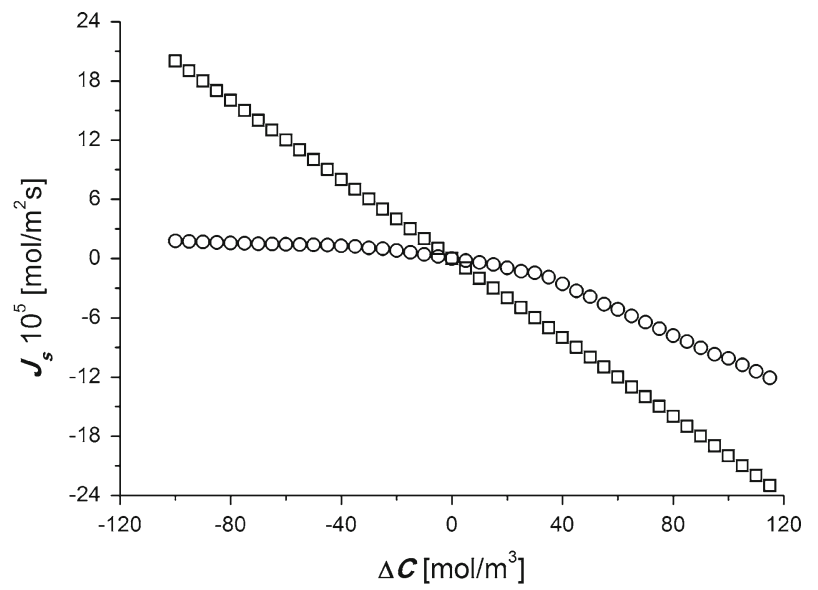

Fig. 4 Glucose concentration difference $\left(\Delta C=C_{h}-C_{l}\right)$ dependence of diffusive solute fluxes $\left(J_{S}, J_{s S}\right)$ for the single-membrane system: $J_{s}=f(\Delta C)$ in homogeneity solutions conditions (open square); $J_{s s}=f(\Delta C$ ) in concentration polarization conditions (open circle) for $\Delta P=0$

2005). These instabilities are caused by sufficiently large gradients of solution's densities in CBLs directed in opposition to the gravity vector (Jasik-Ślęzak et al. 2011).

In Fig. 5, the characteristic of $J_{s a}=f\left(\bar{C} J_{v}\right)_{\Delta \pi=0}$ is shown. In this case, $J_{s a}$ was obtained for $\bar{C}$ and $J_{v}$ presented in Fig. 4 . $J_{s a}$ is linearly dependent on $\bar{C} J_{v}$. The study of $J_{s a}$, conducted under conditions of homogeneity of solutions separated by the membrane and concentration polarization conditions for the configurations A and B of the single-membrane system. The obtained experimental results fit within a $5 \%$ measurement error range.

Taking into consideration the experimentally determined transport parameters $\left(L_{p}, \sigma, \omega\right)$ of the Nephrophan membrane, the volume and solute fluxes $J_{v h}, J_{v s}, J_{s s}$ and $J_{s a}$ shown in Figs. 2, 3, 4, 5 and Eqs. (5-8), the coefficients $\zeta_{p}, \zeta_{v}, \zeta_{s}$ and $\zeta_{a}$ were calculated for aqueous solutions of glucose. Considering results of $J_{v h}$ study shown in Fig. 2, in Eq. (5), 


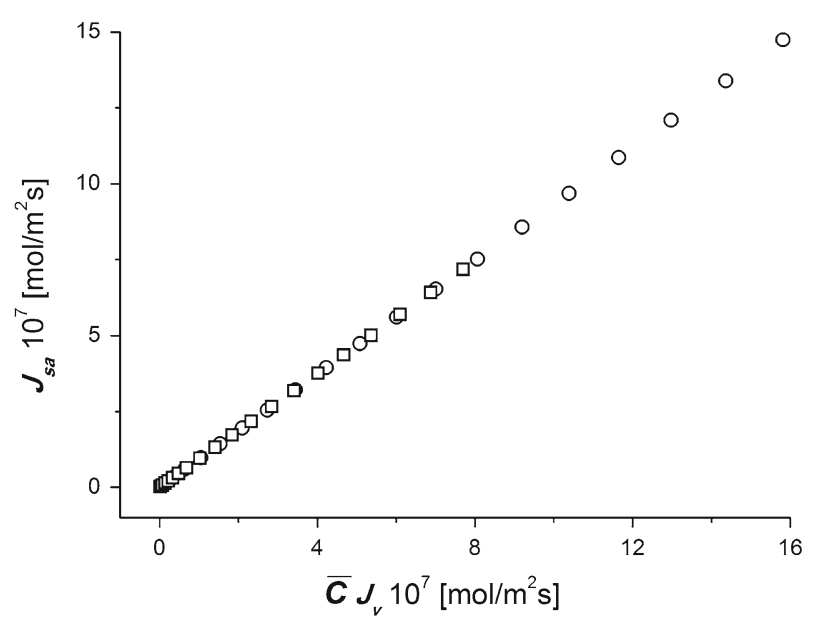

Fig. 5 The advective solute flux $\left(J_{s a}\right)$ through the membrane for solutions with (open circle), and without (open square) mechanical stirring as a function of product of $\bar{C} J_{v}$ for $\Delta \pi=C_{h} C_{l}=0\left(C_{h}=C_{l} \neq 0\right)$

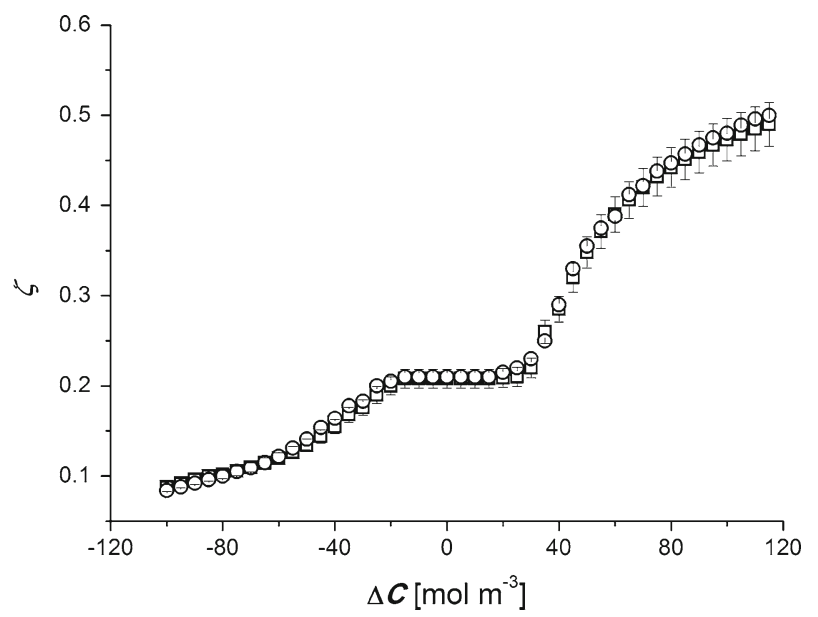

Fig. 6 The concentration polarization coefficients $\zeta_{v}$ (open square) and $\zeta_{s}$ (open circle) as functions of difference of glucose concentrations $(\Delta C)$

we obtain $\zeta_{p}=1=$ const. This means that the value of coefficient $\zeta_{p}$ is independent of solution concentration and membrane system configurations. Taking into account volume $J_{v s}$ and solute $J_{s s}$ fluxes presented in Figs. 3 and 4 in Eqs. (9) and (10), the characteristics $\zeta_{v}=f(\Delta C)$ and $\zeta_{s}=f(\Delta C)$ were obtained and are shown in Fig. 6. The difference between $\zeta_{v}$ and $\zeta_{s}$ is located within $6 \%$ measurement error range. Therefore, it can be written that $\zeta_{v}=\zeta_{s}=\zeta$. Taking into account the volume flux $J_{s a}$ presented in Fig. 5 in Eq. (8) gives $\zeta_{a}=1$. This means that value of this coefficient is independent of glucose concentration and membrane system configuration.

The coefficients $\zeta_{v}$ and $\zeta_{s}$ as functions of difference of glucose concentration $(\Delta C)$ are presented in Fig. 6. The presented curves were obtained for configurations A and B of the membrane system. The configuration $\mathrm{A}$ is the part of the relation $\zeta_{v}=f(\Delta C)$ and 


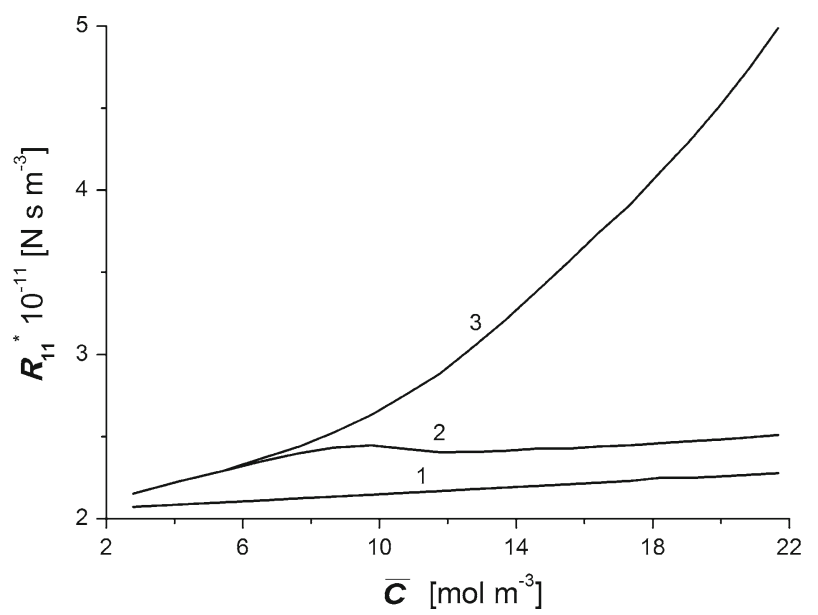

Fig. 7 The graphic illustration of dependence $R_{11}^{*}=f(\bar{C})$ for aqueous glucose solutions in conditions of concentration polarization for the configuration A (graph 3), and B (graph 2) of the membrane system. The straight line 1 illustrates the dependence $R_{11}=f(\bar{C})$ in conditions of homogeneous solutions separated by the membrane. The values of coefficients, $R_{11}^{*}$, were calculated on the basis of Eq. (11a) and $R_{11}$ on the basis of Eq. (11b)

$\zeta_{s}=f(\Delta C)$, obtained for $\Delta C<0$, while the relation $\zeta_{v}=f(\Delta C)$ and $\zeta_{s}=f(\Delta C)$, obtained for $\Delta C>0$ corresponds to the configuration B. Data presented in Fig. 6 indicate that values $\zeta_{v}$ and $\zeta_{s}$, for $\Delta C>15 \mathrm{~mol} \mathrm{~m}^{-3}$ are different for configurations $\mathrm{A}$ and $\mathrm{B}$, which means they are different for both gravitational directions of the membrane transport. This is connected with diversified hydrodynamic characters of concentration boundary layers. For the whole range of $\Delta C$, the layers in the configuration $\mathrm{A}$ are hydrodynamically stable, while in configuration $\mathrm{B}$, convectional instabilities for $\Delta C>15 \mathrm{~mol} \mathrm{~m}^{-3}$ cause the destruction of CBLs. This is the reason for the higher values of $\zeta_{v}$ and $\zeta_{s}$ in configuration B comparing to values of $\zeta_{v}$ and $\zeta_{s}$ in configuration $\mathrm{A}$. The higher value of coefficients $\zeta_{v}$ and $\zeta_{s}$ in the configuration $\mathrm{B}$ are caused by the fact that, in the case of the solutions with higher density above the membrane, the phenomenon of convection causes greater flows through the membrane. It means that for $\Delta C$ in the range $-15 \leq \Delta C \leq 15 \mathrm{molm}^{-3}$, values $\zeta_{v}$ and $\zeta_{s}$ do not depend significantly on the configuration of the membrane system. The condition $\Delta C \leq 15 \mathrm{~mol} \mathrm{~m}^{-3}$ can be written also as $\bar{C} \leq 5.41 \mathrm{~mol} \mathrm{~m}^{-3}$.

Taking into consideration the experimentally determined transport parameters of the Nephrophane membrane $\left(L_{p}, \sigma, \omega\right), \zeta_{v}$ and $\zeta_{s}$ shown in Fig. 6, Eqs. (11a) and (11b), and assuming $\zeta_{p}=\zeta_{a}=1$, the calculations of values of resistance coefficients: $R_{11}, R_{12}=$ $R_{21}, R_{22}, R_{11}^{*}, R_{12}^{*}, R_{21}^{*}$ and $R_{22}^{*}$ were carried out for aqueous glucose solutions for both the configurations of the membrane system.

In order to calculate the coefficients of the matrix $\left[R^{*}\right]$ the transport parameters of the Kedem and Katchalsky's formalism were used. As is already, known (Katchalsky and Curran $1965)$ this formalism contains coefficients of hydraulic permeability $\left(L_{p}\right)$, reflection $(\sigma)$ and diffusive permeability $(\omega)$ determined for homogeneous solutions separated by a membrane.

The coefficients $R_{11}^{*}, R_{12}^{*}, R_{21}^{*}, R_{22}^{*}$ and $R_{11}, R_{12}=R_{21}, R_{22}$ as functions of mean concentration in the membrane $(\bar{C})$ are presented in Figs. 7, 8 and 9. From the course of graphs 2 and 3 presented in Fig. 7, it results that, for $\bar{C} \leq 5.41 \mathrm{molm}^{-3}$, the value of coefficient $R_{11}^{*}$ depends on concentration $\bar{C}$ and does not depend on configuration of the 


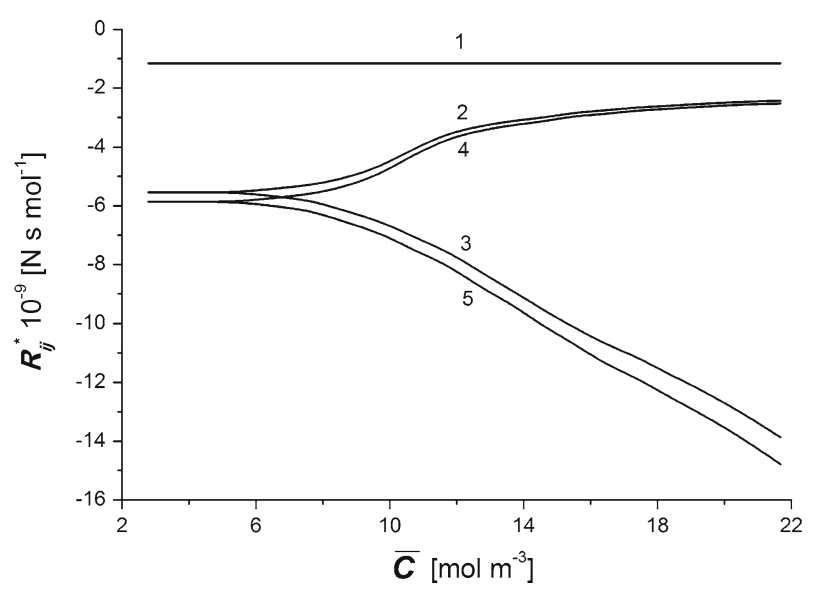

Fig. 8 The graphic illustration of dependence $R_{i j}^{*}=f(\bar{C})$ for aqueous glucose solutions in conditions of concentration polarization for the configuration A (graphs 3 and 5) and B (graphs 2 and 4) of the membrane system. Graphs 2 and 3 were obtained for $R_{12}^{*}=f(\bar{C})$, and graphs 4 and 5 for $R_{21}^{*}=f(\bar{C})$. The straight line 1 illustrates the dependence $R_{12}=R_{21}=f(\bar{C})$ in conditions of homogeneous solutions separated by the membrane. The values of coefficients $R_{12}^{*}$ and $R_{21}^{*}$ were calculated on the basis of Eq. (11a), and $R_{12}=R_{21}$ on the basis of Eq. (11b)

membrane system. For $\bar{C}>5.41 \mathrm{molm}^{-3}$, the value of coefficient $R_{11}^{*}$ depends on both $\bar{C}$ and configuration of the membrane system. The point $R_{11}^{*}=2.4 \times 10^{9} \mathrm{~N} \mathrm{~s} \mathrm{~m}^{-3}$ with $\bar{C}=5.41 \mathrm{~mol} \mathrm{~m}^{-3}$ is the last joint point of curves 2 and 3 . Besides, for configuration A, the values $R_{11}^{*}$ (curve 3 ) for the same value of $\bar{C}$ are greater than those for configuration $\mathrm{B}$ (curve 2).

From Fig. 8, it results that for $\bar{C} \leq 5.41 \mathrm{~mol} \mathrm{~m}^{-3}$, the values of coefficients $R_{12}^{*}$ and $R_{21}^{*}$ (and $R_{21}^{*} \neq R_{12}^{*}$ ) do not depend on concentration $\bar{C}$ and the configuration of the membrane system, which is illustrated by curves 2 or 4 (for configuration B) and 3 or 5 (for configuration A). From this figure, it also results that for the same $\bar{C}$, the values of coefficients $R_{12}^{*}$ and $R_{21}^{*}$ for configuration $\mathrm{A}$ are lower than those for configuration $\mathrm{B}$. Besides, the point $R_{12}^{*}=$ $-5.22 \times 10^{9} \mathrm{~N} \mathrm{~s} \mathrm{~mol}^{-1}$ with $\bar{C}=5.41 \mathrm{~mol} \mathrm{~m}^{-3}$ is the last joint point of curves 2 and 3 , whereas point $R_{21}^{*}=-4.94 \times 10^{9} \mathrm{~N} \mathrm{~s} \mathrm{~mol}^{-1}$ with $\bar{C}=5.41 \mathrm{~mol} \mathrm{~m}^{-3}$ is the last joint point of curves 4 and 5 .

The curves 2 and 3 shown in Fig. 9 demonstrate that the value of coefficient $R_{22}^{*}$ decreases hyperbolically with increase of $\bar{C}$ in whole range of studied glucose concentrations, but for $\bar{C} \leq 5.41 \mathrm{molm}^{-3}, R_{22}^{*}$ values do not depend on the membrane configuration. For $\bar{C}>5.41 \mathrm{~mol} \mathrm{~m}^{-3}$, the value of coefficient $R_{22}^{*}$ depends on both concentration $\bar{C}$ and the configuration of the membrane system. The point $R_{22}^{*}=0.975 \times 10^{9} \mathrm{~N} \mathrm{~s} \mathrm{~m}^{3} \mathrm{~mol}^{-2}$ with $\bar{C}=5.41 \mathrm{~mol} \mathrm{~m}^{-3}$ is the last joint point of curves 2 and 3 . Besides, for configuration A, the values of $R_{22}^{*}$, for the same concentration $\bar{C}$ are greater than those for configuration $\mathrm{B}$. The last joint points of curves shown in Figs. 7, 8 and 9 can be treated as bifurcation points. This means that transition through bifurcation point and attainment by $R_{11}^{*}, R_{12}^{*}, R_{21}^{*}$ and $R_{22}^{*}$ values belonging to curves 2 and 3 illustrate the possible choice between convective (configuration B) and non-convective (configuration A) states of the membrane system.

From Figs. 7, 8 and 9 it results that the values of coefficients $R_{11}, R_{12}=R_{21}$ and $R_{22}$ do not depend on the configuration of the membrane system. The straight line shown in Fig. 7 demonstrates that the value of coefficient $R_{11}$ linearly increases with increase of 


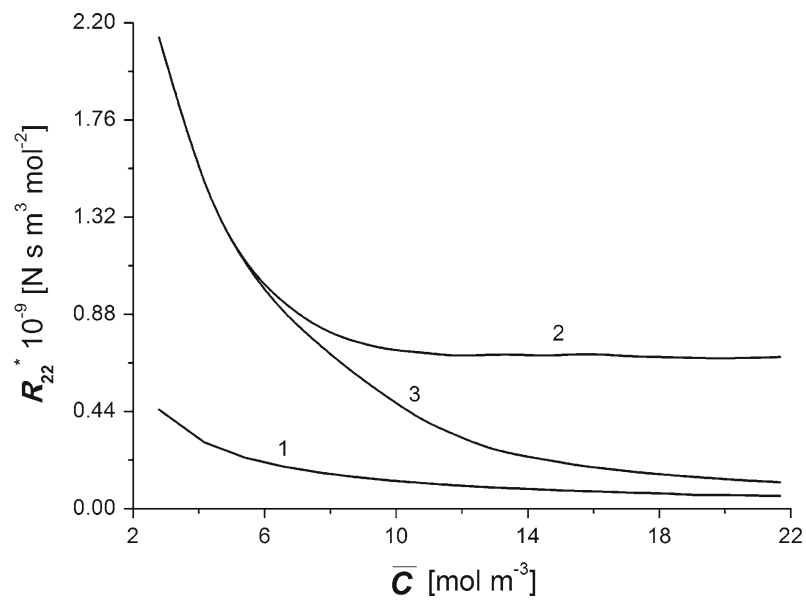

Fig. 9 The graphic illustration of dependence $R_{22}^{*}=f(\bar{C})$ for aqueous glucose solutions in conditions of concentration polarization for the configuration A (graph 2) and B (graph 3) of the membrane system. The curve 1 illustrates the dependence $R_{22}=f(\bar{C})$ in conditions of homogeneous solutions separated by the membrane. The values of coefficients $R_{22}^{*}$ were calculated on the basis of Eq. (11a), and $R_{22}$ on the basis of Eq. (11b)

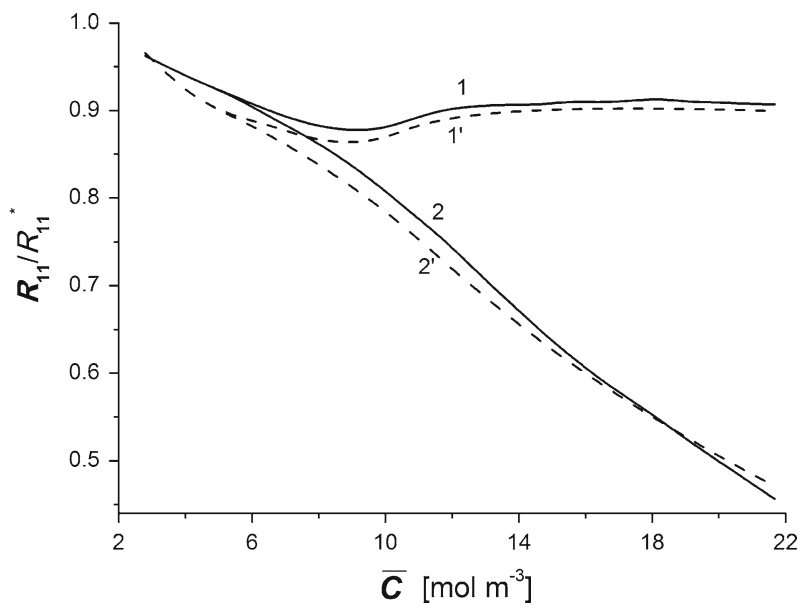

Fig. 10 The graphic illustration of dependence $R_{11} / R_{11}^{*}=f(\bar{C})$ for the configuration A (graphs 2 and $2^{\prime}$ ) and $\mathrm{B}$ (graphs 1 and $1^{\prime}$ ) of the membrane system. Curves 1 and 2 were calculated on the basis of Eq. (13), curve $2^{\prime}$ on the basis of Eq. (20), and curve $1^{\prime}$ on the basis of Eq. (24)

mean concentration of glucose in the membrane $\bar{C}$. The value of coefficient $R_{11}$ does not depend on mean glucose concentration in the membrane, and amounts to $R_{12}=R_{21}=$ $-1.16 \times 10^{9} \mathrm{Ns} \mathrm{mol}^{-1}$, which is illustrated by straight line in Fig. 8. The data illustrated by curve 1 in Fig. 9 show that the value of coefficient $R_{22}$ hyperbolically decreases with the increase of concentration $\bar{C}$.

In order to demonstrate the influence of concentration polarization on the resistance coefficients of the membrane $\left(R_{i j}\right)$, the ratios $R_{11} / R_{11}^{*}, R_{12} / R_{12}^{*}, R_{21} / R_{21}^{*}$ and $R_{22} / R_{22}^{*}$ are shown in Figs. 10, 11 and 12 as functions of concentration $C$. In Fig. 10, full line illustrating the dependence $R_{11} / R_{11}^{*}=f(\bar{C})$, calculated on the basis of Eq. (13), was shown for the 


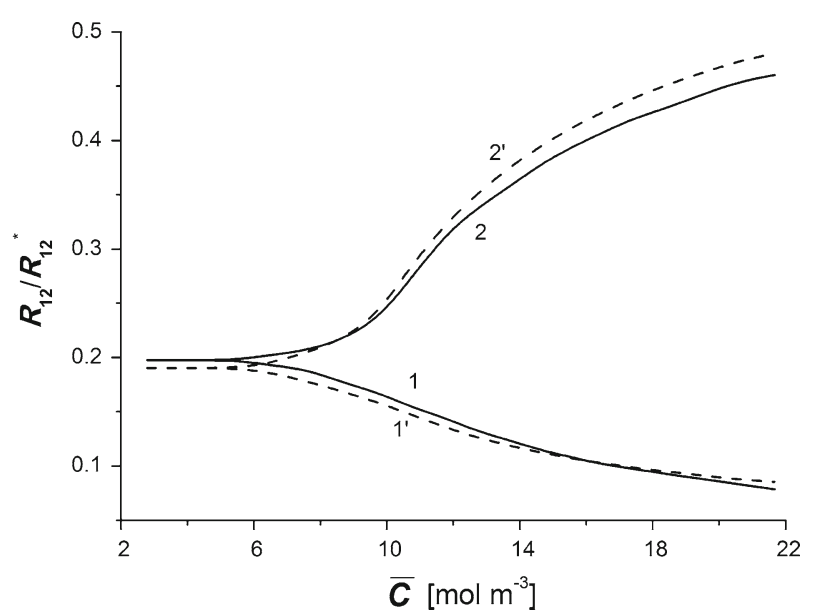

Fig. 11 The graphic illustration of dependence $R_{12} / R_{12}^{*}=f(\bar{C})$ for the configuration A (graphs 1 and $1^{\prime}$ ) and B (graphs 2 and $2^{\prime}$ ) of the membrane system. Curves 1 and 2 were calculated on the basis of Eq. (14), curve $2^{\prime}$ on the basis of Eq. (21), and curve $1^{\prime}$ on the basis of Eq. (25)

configuration A (curve 2) and for configuration B (curve 1). From this figure, it results that for $\bar{C} \leq 5.41 \mathrm{~mol} \mathrm{~m}^{-3}$, the ratio $R_{11} / R_{11}^{*}$ does not depend on $\bar{C}$ and for $\bar{C}>5.41 \mathrm{~mol} \mathrm{~m}^{-3}$, it depends on concentrations of solutions separated by the membrane and on the configuration of the membrane system. The point $R_{11} / R_{11}^{*}=0.87$ with $\bar{C}=5.41 \mathrm{~mol} \mathrm{~m}^{-3}$ is the last joint point of curves 1 and 2. From Fig. 10, it results that for $\bar{C}>5.41 \mathrm{~mol} \mathrm{~m}^{-3}$, the values of ratio $R_{11} / R_{11}^{*}$ for the configuration $\mathrm{A}$ are lower in comparison to those of $R_{11} / R_{11}^{*}$, for the configuration $\mathrm{B}$.

The dependencies $R_{12} / R_{12}^{*}=f(\bar{C})$, for the configuration A (curve 2 ) and the configuration B (curve 1), calculated on the basis of Eq. (14), are demonstrated in Fig. 11. From this figure, it results that for $\bar{C} \leq 5.41 \mathrm{molm}^{-3}$, the values of ratio $R_{12} / R_{12}^{*}$ do not depend, and for $\bar{C}>5.41 \mathrm{~mol} \mathrm{~m}^{-3}$ they depend on the concentrations of solutions separated by the membrane and the configuration of the membrane system. The point $R_{12} / R_{12}^{*}=0.22$ with $\bar{C}$ $=5.41 \mathrm{~mol} \mathrm{~m}^{-3}$ is the last joint point of curves 1 and 2. Besides, from Fig. 11, it results that for $\bar{C}>5.41 \mathrm{~mol} \mathrm{~m}^{-3}$, the values of ratio $R_{12} / R_{12}^{*}$ for the configuration A are lower than the values of this ratio for the configuration $\mathrm{B}$.

The dependencies of $R_{21} / R_{21}^{*}=f(\bar{C})$ and $R_{22} / R_{22}^{*}=f(\bar{C})$ for the configuration $\mathrm{A}$ (curves 2 and 4 ) and the configuration B (curves 1 and 3), calculated on the basis of Eqs. (15) and (16) are shown in Fig. 12. From this figure, it results that for $\bar{C} \leq 5.41 \mathrm{~mol} \mathrm{~m}^{-3}$, the ratios $R_{21} / R_{21}^{*}$ and $R_{22} / R_{22}^{*}$ do not depend and for $\bar{C}>5.41 \mathrm{~mol} \mathrm{~m}^{-3}$, they depend on glucose concentrations of solutions separated by the membrane and the configuration of the membrane system. The points $R_{21} / R_{21}^{*}=0.24$ with $\bar{C}=5.41 \mathrm{~mol} \mathrm{~m}^{-3}$ (for curves 1 and 2) and $R_{22} / R_{22}^{*}=0.21$ with $\bar{C}=5.41 \mathrm{~mol} \mathrm{~m}^{-3}$ (for curves 3 and 4 ) are the last joint points of these curves. Besides, from Fig. 12, it results that for $\bar{C}>5.41 \mathrm{~mol} \mathrm{~m}^{-3}$ the values of ratios $R_{21} / R_{21}^{*}$ and $R_{22} / R_{22}^{*}$ for the configuration $\mathrm{A}$ are lower than those for the configuration $\mathrm{B}$. The last joint points of curves for the configurations A and B of the membrane system shown in Fig. 12, similarly as the last joint points for graphs shown in Figs. 7, 8, 9, 10 and 11 can be treated as bifurcation points.

In order to show the relation between the configurations B and A of the membrane system the corresponding ratios of resistance coefficients $R_{11}^{*}, R_{12}^{*}, R_{21}^{*}$ or $R_{22}^{*}$ for the configuration $\mathrm{B}$ and $\mathrm{A}$ of the membrane system were calculated on the basis of expression: 


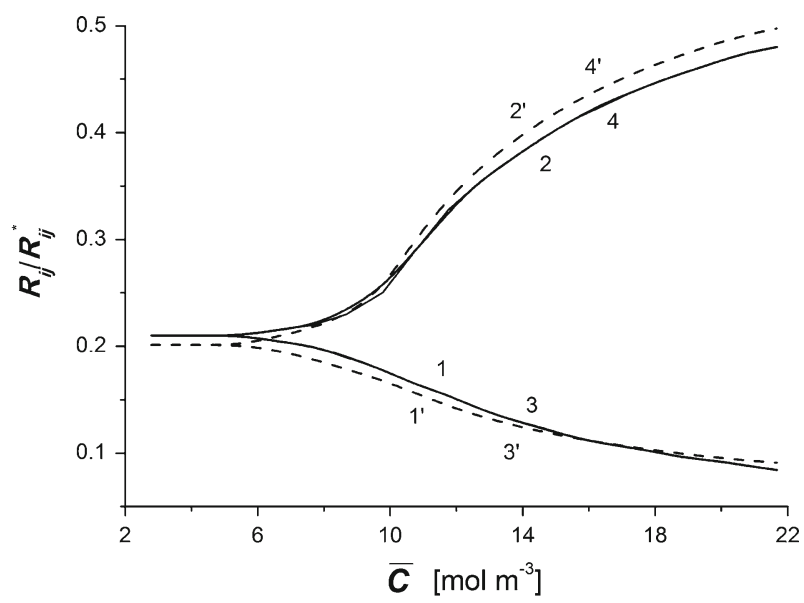

Fig. 12 The graphic illustration of dependence $R_{i j} / R_{i j}^{*}=f(\bar{C})$ for the configuration A (graphs $1,1^{\prime}, 3$ and $3^{\prime}$ ) and B (graphs 2, 2', 4 and $4^{\prime}$ ) of the membrane system. Graphs 1, 2, $1^{\prime}$ and $2^{\prime}$ were obtained for $R_{21} /$ $R_{21}^{*}=f(\bar{C})$; and graphs $3,4,3^{\prime}$ and 4 for $R_{22} / R_{22}^{*}=f(\bar{C})$. Curves 1 and 2 were calculated on the basis of Eq. (15), curves 3 and 4 on the basis of Eq. (16), curves $1^{\prime}$ and $3^{\prime}$ on the basis of Eq. (22), and curves $2^{\prime}$ and $4^{\prime \prime}$ on the basis of Eq. (26)

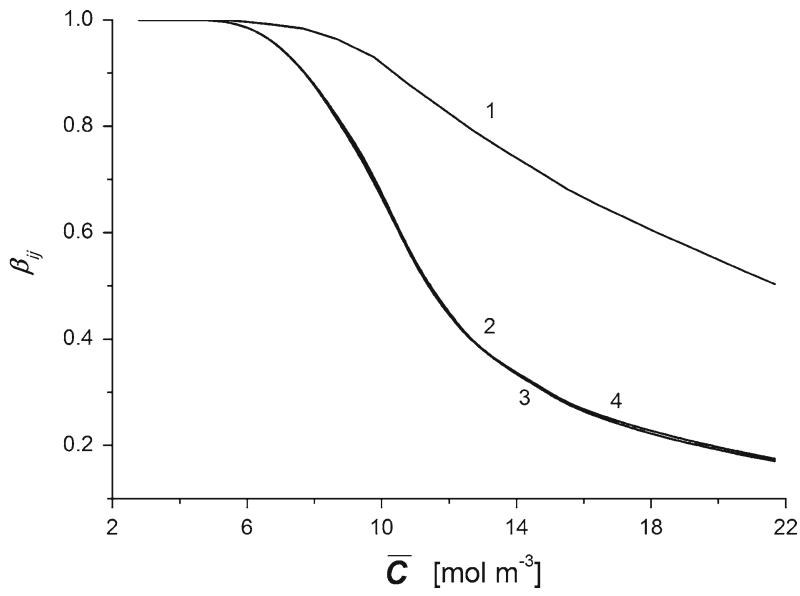

Fig. 13 The graphic illustration of dependence $\beta_{i j}=f(\bar{C})$ for aqueous glucose solutions in conditions of concentration polarization. The graph 1 illustrates the dependence $\beta_{11}=f(\bar{C})$, graph 2 the dependence $\beta_{12}=f(\bar{C})$, graph 3 the dependence $\beta_{21}=f(\bar{C})$, and graph 4 the dependence $\beta_{22}=f(\bar{C})$. The values of coefficient $\beta_{i j}$ were calculated on the basis of Eq. (18)

$$
\beta_{i k}=\frac{\left(R_{i k}^{*}\right)_{B}}{\left(R_{i k}^{*}\right)_{A}}, i \neq k=1,2
$$

The results of calculations on the basis of Eq. (18) shown in Fig. 13 illustrate the dependencies of $\beta_{11}=f(\bar{C})$ (curve 1), $\beta_{12}=f(\bar{C})$ (curve 2), $\beta_{21}=f(\bar{C})$ (curve 3) and $\beta_{22}=f(\bar{C}$ ) (curve 4). From Fig. 13, it results that for $\bar{C} \leq 5.41 \mathrm{~mol} \mathrm{~m}^{-3}$, the values of coefficients $\beta_{11}, \beta_{12}$ and $\beta_{22}$ do not depend on concentration of solutions separated by the membrane and the configuration of the membrane system. The values of these coefficients amount to 
$\beta_{11}=\beta_{12}=\beta_{21}=\beta_{22}=1$ and the last joint point of curves is for $\bar{C}=5.41 \mathrm{~mol} \mathrm{~m}^{-3}$, which corresponds to bifurcation point resulting from the curves 2 and 3 shown in Figs. 7, 8 and 9 . For $\bar{C}>5.41 \mathrm{molm}^{-3}$, the values of coefficients $\beta_{11}, \beta_{12}, \beta_{21}$ and $\beta_{22}$ depend on solutions concentrations separated by the membrane. Comparing these characteristics, we can state that for the same values of concentration $\bar{C}$, the coefficients $\beta_{12}$ and $\beta_{22}$ are equal and are lower than $\beta_{11}$.

Moreover, the results of experimental studies for Nephrophan membrane and aqueous solutions of glucose presented in this article suggest that with sufficient approximation, we can assume the following conditions $\zeta_{p}=\zeta_{a}=1$ and $\zeta_{v}=\zeta_{s}=\zeta$. In a previous article (Ślęzak et al. 2005) it was shown that the coefficient $\zeta_{i}$ can be presented in the form:

$$
\zeta_{i}=D_{i}\left(D_{i}+2 R T \omega \delta_{i}\right)^{-1}
$$

where $D_{i}$ is the diffusion coefficient, $R T$ is the product of gas constant and thermodynamic temperature and $\delta_{i}$ is the thickness of the concentration boundary layer. For the conditions of diffusion and $i=d$, and for the convection $i=k$.

Taking into account Eq. (19) and conditions $\zeta_{p}=\zeta_{a}=1$ and $\zeta_{v}=\zeta_{s}=\zeta$ in Eqs. (13)-(16), we obtain

$$
\begin{aligned}
\frac{R_{11}}{R_{11}^{*}} & =\frac{D_{i}\left[\bar{C}(1-\sigma)^{2} L_{p}+\omega\right]}{D_{i}\left[\bar{C}(1-\sigma)^{2} L_{p}+\omega\right]+2 R T \omega \delta_{i} \bar{C}(1-\sigma) L_{p}} \\
\frac{R_{12}}{R_{12}^{*}} & =\frac{D_{i}(1-\sigma)}{D_{i}(1-\sigma)+2 R T \omega \delta_{i}} \\
\frac{R_{21}}{R_{21}^{*}} & =\frac{D_{i}}{D_{i}+2 R T \omega \delta_{i}}=\frac{R_{22}}{R_{22}^{*}}
\end{aligned}
$$

In non-convective conditions thicknesses of CBLs $\left(\delta_{d}\right)$ are larger than in conditions of natural convection $\left(\delta_{k}\right)$. Thicknesses $\delta_{d}$ and $\delta_{k}$ can be determined by optical methods (Dworecki 1995) or by measurements of volume flows (Ślęzak et al. 2010; Jasik-Ślęzak et al. 2011). Moreover, in diffusion conditions, the value of diffusion coefficient $\left(D_{d}\right)$ is constant and independent of concentration of dilute solutions of non-electrolytes. In turn, in convective conditions, coefficient $D_{k}\left(D_{k}>D_{d}\right)$ can be expressed using the following equation (Ślęzak et al. 2010):

$$
D_{k}=\frac{g \alpha_{C} \delta_{k}^{3}\left(J_{v}-J_{v s}\right)}{2 L_{p} \sigma R T \nu R_{C}}
$$

Taking into account Eq. (23) in Eqs. (21) and (22) in convective conditions we obtain

$$
\begin{aligned}
\frac{R_{11}}{R_{11}^{*}} & =\frac{g \alpha_{C} \delta_{k}^{2}\left(J_{v}-J_{v s}\right)\left[\bar{C}(1-\sigma)^{2} L_{p}+\omega\right]}{g \alpha_{C} \delta_{k}^{2}\left(J_{v}-J_{v s}\right)\left[\bar{C}(1-\sigma)^{2} L_{p}+\omega\right]+4(R T)^{2} L_{p}^{2} \nu R_{C} \omega \bar{C} \sigma(1-\sigma)} \\
\frac{R_{12}}{R_{12}^{*}} & =\frac{g \alpha_{C} \delta_{k}^{2}(1-\sigma)\left(J_{v}-J_{v s}\right)}{g \alpha_{C} \delta_{k}^{2}(1-\sigma)\left(J_{v}-J_{v s}\right)+4(R T)^{2} L_{p} \omega \sigma R_{C}} \\
\frac{R_{21}}{R_{21}^{*}} & =\frac{g \alpha_{C} \delta_{k}^{2}\left(J_{v}-J_{v s}\right)}{g \alpha_{C} \delta_{k}^{2}\left(J_{v}-J_{v s}\right)+4(R T)^{2} L_{p} \omega \sigma v R_{C}}=\frac{R_{22}}{R_{22}^{*}}
\end{aligned}
$$

For example, we calculate the ratios $R_{11} / R_{11}^{*}=f(\bar{C}), R_{12} / R_{12}^{*}=f(\bar{C}), R_{21} / R_{21}^{*}=f(\bar{C})$ and $R_{22} / R_{22}^{*}=f(\bar{C})$ on the basis of Eqs. (20)-(22) as appropriate for non-convective and on the basis of Eqs. (24)-(26) - for convective conditions. The following data were used: $\mathrm{g}=9.81 \mathrm{~m} \mathrm{~s}^{-2}, \alpha_{C}=6.01 \times 10^{-5} \mathrm{~m}^{3} \mathrm{~mol}^{-1}, \omega=0.8 \times 10^{-9} \mathrm{~mol} \mathrm{~N}^{-1} \mathrm{~s}^{-1}, \sigma=0.068$, 
$L_{p}=4.9 \times 10^{-12} \mathrm{~m}^{3} \mathrm{~N}^{-1} \mathrm{~s}^{-1}, v=1.012 \times 10^{-6} \mathrm{~m}^{2} \mathrm{~s}^{-1}, D_{d}=\mathrm{const}=0.69 \times 10^{-9} \mathrm{~m}^{2} \mathrm{~s}^{-1}$ (for non-convective conditions), dependencies $J_{v}=f(\bar{C})$ and $J_{v s}=f(\bar{C})$ shown in Fig. 3 and dependencies of CBLs thicknesses $\delta_{d}$ and $\delta_{k}$ as functions of mean concentration in the membrane presented in (Ślęzak et al. 2010; Jasik-Ślęzak et al. 2011). The dependencies of $R_{11} / R_{11}^{*}=f(\bar{C})$ calculated on the basis of Eqs. (20) and (24) for the non-convective $\left(2^{\prime}\right)$ and convective $\left(1^{\prime}\right)$ conditions are shown in Fig. 10. The dependencies of $R_{12} / R_{12}^{*}=f(\bar{C})$ calculated on the basis of Eqs. (21) and (25) for the non-convective $\left(1^{\prime}\right)$ and convective $\left(2^{\prime}\right)$ conditions are shown in Fig. 11. The dependencies of $R_{21} / R_{21}^{*}=R_{22} / R_{22}^{*}=f(\bar{C})$ calculated on the basis of Eqs. (22) and (26) for the non-convective ( $\left.1^{\prime}\right)$ and convective $\left(2^{\prime}\right)$ conditions are shown in Fig. 12. From these figures it results that adequate resistance coefficients ratios obtained from calculations on the basis of Eqs. (13)-(16) and (20)-(22), (24)-(26) correspondingly are similar.

As per the results from presented data, the concentration polarization of the membrane causes increase of resistance coefficients $R_{i j}^{*}$ in comparison with homogeneous conditions, while appearance of gravitational convection in chambers of the membrane system is the cause of decrease of the level of concentration polarization of the membrane and decreases of resistance coefficients values $R_{i j}^{*}$. Besides, as per results from Figs. 7, 8, 9, 10, 11, 12 and 13 , increase of mean concentration in the membrane (with constant lower concentration $C_{l}$ ) is connected with increase of difference between the configurations $\mathrm{A}$ and $\mathrm{B}$ of the membrane system. The increase of difference between the configurations A and B of the membrane system is caused by the growing intensity of gravitational stirring of solutions. As can be noticed also in these figures, increase of mean concentration in the membrane causes that the differences between presented parameters of the membrane system for the configuration B and the membrane system with homogeneous conditions are lower. This is due to the more intensive gravitational stirring of solutions in the case of the configuration $\mathrm{B}$ for greater values of mean concentration in the membrane with constant concentration $C_{l}$.

\section{Conclusions}

From the above presented study, the following results are obtained:

1. Peusner's Network Thermodynamics is one of alternative ways of membrane transport description in conditions of both homogeneous solutions separated by membrane and concentration polarization.

2. The resistance coefficients $R_{11}^{*}, R_{12}^{*}, R_{21}^{*}$ and $R_{22}^{*}$ are nonlinearly dependent on solutions concentrations separated by the membrane, and it was found that the threshold value of concentration exists, above which the resistance coefficients $R_{11}^{*}, R_{12}^{*}, R_{21}^{*}$ and $R_{22}^{*}$ depend on the configuration of the membrane system.

3. The values of coefficients $R_{11}^{*}, R_{12}^{*}, R_{21}^{*}$ and $R_{22}^{*}$ in non-convective states are greater than those in convective states. Besides increase of mean concentration in the membrane with constant lower concentration causes increase of difference between corresponding resistance coefficients for the configuration $\mathrm{A}$ and $\mathrm{B}$ of the membrane system. This is caused by more intensive gravitational stirring of solutions in the membrane system in the configuration $\mathrm{B}$.

Acknowledgements This study was supported by the Ministry of Science and Higher Education under Grant No. BS/PB -622/3010/2011/P. 
Open Access This article is distributed under the terms of the Creative Commons Attribution License which permits any use, distribution, and reproduction in any medium, provided the original author(s) and the source are credited.

\section{References}

Barry, P.H., Diamond, J.M.: Effects of unstirred layers on membrane phenomena. Physiol. Rev. 64, 763-872 (1984)

Demirel, Y.: Nonequilibrium Thermodynamics, Transport and Rate Processes in Physical and Biological Systems. Elsevier, Amsterdam (2002)

Dworecki, K.: Interferometric investigations of near-membrane diffusion layers. J. Biol. Phys. 21, 37-49 (1995)

Dworecki, K., Ślęzak, A., Ornal-Wąsik, B., Wąsik, S.: Effect of hydrodynamic instabilities on solute transport in a membrane system. J. Membr. Sci. 265, 94-100 (2005)

Ewing, G.W.: Instrumental methods of chemical analysis. McGraw-Hill, New York (1985)

Ginzburg B., Z., Katchalsky, A.: The frictional coefficients of the flows of non- electrolytes through artificial membranes. J. Gen. Physiol. 47, 403-418 (1963)

Grzegorczyn, S., Jasik-Ślęzak, J., Michalska-Małecka, K., Ślęzak, A.: Transport of non-electrolyte solutions through membrane with concentration polarization. Gen. Physiol. Biophys. 27, 315-321 (2008)

Imai, Y.: Network thermodynamics: analysis and synthesis of membrane transport system. Japan. J. Physiol. 46, 187-199 (1996)

Imai, Y.: Graphic modeling of epithelial transport system: causality of dissipation. BioSystems 70, 9-19 (2003)

Jasik-Ślęzak, J., Ślęzak, A.: Thermodynamical description of the concentration polarization in a membrane transport of nonelectrolyte solution. Polym. Med. 40, 49-55 (2010)

Jasik-Ślęzak, J., Olszówka, K.M., Ślęzak, A.: Estimation of thickness of concentration boundary layers by osmotic volume flux determination. Gen. Physiol. Biophys. 30, 186-195 (2011)

Kargol, A.: Effect of boundary layers on reverse osmosis through a horizontal membrane. J. Membr. Sci. 159, 177-184 (1999)

Kargol, M., Kargol, A.: Membrane transport generated by the osmotic and hydrostatic pressure. Correlation relation for parameters $1_{p}, \sigma$ and $\omega$. J. Biol. Phys. 26, 307-320 (2000)

Kargol, A.: Modified Kedem-Katchalsky equations and their application. J. Membr. Sci. 174, 43-53 (2000)

Katchalsky, A., Curran P., F.: Nonequilibrium Thermodynamics in Biophysics. Harvard, Cambridge (1965)

Klinkman, H., Holtz, M., Willgerodt, W., Wilke, G., Schoenfelder, D.: Nephrophan-eine neue dialysemembranes. Z. Urol. 4, 285-292 (1969)

Larchet, C., Nouri, S., Auclair, B., Dammak, L., Nikonenko, V.: Application of chronopotentiometry to determine the thickness of diffusion layer adjacent to an ion-exchange membrane under natural convection. Adv. Colloid Interface Sci. 139, 45-61 (2008)

Meixner, J.: Network theory in its realation to thermodynamics. In: Fox, J. (ed.) Proceedings of the Symposium on Generalized Networks, pp. 13-25. Wiley, New York (1966)

Mikulecky, D.: The circle that never ends: can complexity be made simple? In: Bonvchev, D.D., Rouvaray, D. (eds.) Complexity in Chemistry, Biology and Ecology, pp. 97-153. Springer, Berlin (2005)

Mishchuk, N.A.: Concentration polarization of interface and non-linear electrokinetic phenomena. Adv. Colloid Interface Sci. 160, 16-39 (2010)

Newman, S.A., Forgacs, G. : Complexity and self-organization in biological development and evolution. In: Bonvchev, D.D., Rouvaray, D. (eds.) Complexity in Chemistry, Biology and Ecology, pp. 49-96. Springer, Berlin (2005)

Nicolis, G., Prigogine, I.: Self-Organization in Nonequilibrium Systems. From Dissipative Structures to Order Through Fluctuactions. Wiley, New York (1977)

Oster, G.F., Perelson, A., Katchalsky, A.: Network thermodynamics. Nature 234, 393-399 (1971)

Perelson, A.S.: Network thermodynamics. Biophys. J. 15, 667-685 (1975)

Peusner, L.: The principles of network thermodynamics and biophysical applications. Ph D Thesis, Harvard, Cambridge (1970)

Peusner, L.: Hierarchies of irreversible energy conversion systems: a network thermodynamics approach. I. Linear steady state without storage. J. Theoret. Biol. 102, 7-39 (1983)

Peusner, L.: Hierarchies of irreversible energy conversion systems. II. Network derivation of linear transport equations. J. Theoret. Biol. 115, 319-335 (1985a)

Peusner, L.: Network representation yelding the evolution of Brownian motion with multiple particle interaction. Phys. Rev. 32, 1237-1238 (1985b) 
Peusner, L., Mikulecky, D.C., Bunow, B., Caplan, S.R.: A network thermodynamic approach to hill and King-Altman reaction-diffusion kinetics. J. Chem. Phys. 83, 5559-5566 (1985)

Peusner, L.: Hierarchies of irreversible energy conversion processes. III. Why are Onsager equations reciprocal? The Euclidean geometry of fluctuaction-dissipation space. J. Theoret. Biol. 122, 125-155 (1986a)

Peusner, L.: Studies in Network Thermodynamics. Elsevier, Amsterdam (1986b)

Playtner, H.: Analysis and Design of Engineering Systems. MIT, Cambridge (1961)

Puthenveettil, B.A., Arakeri, J.H.: Convection due to an unstable density difference across a permeable membrane. J. Fluid. Mech. 609, 139-170 (2008)

Puthenveettil, B.A., Gunasegarane, G.S., Agrawal, Y.K., Arakeri, J.H.: Length of near-wall plumes in turbulent convection. J. Fluid Mech. 685, 335-364 (2011)

Ramareddy, G.V., Puthenveettil, B.A.: The Pe $\sim 1$ regime of convection across a horizontal permeable membrane. J. Fluid. Mech. 679, 476-504 (2011)

Rubinstein, I., Zaltzman, B.: Electro-osmotically induced convection at a permselective membrane. Phys. Rev. E 62, 2238-2251 (2000)

Soh, K.C., Hatzimanikatis, V.: Network thermodynamics in the post-genomic era. Curr.Opin. Microbiol. 13, 350-357 (2010)

Ślęzak, A., Dworecki, K., Anderson, J.E.: Gravitational effects on transmembrane flux: the Rayleigh-Taylor convective instability. J. Membr. Sci. 23, 71-81 (1985)

Ślęzak, A., Turczyński, B.: Modiffication of the Kedem-Katchalsky equations. Biophys. Chem. 24, 173178 (1986)

Ślęzak, A.: Irreversible thermodynamic model equations of the transport across a horizontally mounted membrane. Biophys. Chem. 34, 91-102 (1989)

Ślęzak, A., Jasik-Slęzak, J., Wąsik, J., Sierot, A., Pilis, W.: Volume osmotic flows of non-electrolyte solutions through horizontally mounted membrane. Gen. Physiol. Biophys. 21, 115-146 (2002)

Ślęzak, A., Dworecki, K., Ślęzak, I.H., Wąsik, S.: Permeability coefficient model equations of the complex: membrane-concentration boundary layers for ternary nonelectrolyte solutions. J. Membr. Sci. 267, 50-57 (2005)

Ślęzak, A., Grzegorczyn, S., Jasik-Ślęzak, J., Michalska-Małecka, K.: Natural convection as an asymmetrical factor of the transport through porous membrane. Transp. Porous Media 84, 685-698 (2010)

Ślęzak, A.: Application of the network thermodynamics to interpretation of membrane transport: evaluation of the resistance coefficients of the polymeric membrane in polarization concentration conditions. Polim. Med. 41, 43-51 (2011) 